\title{
Inhibition of TGF- $\beta$ with neutralizing antibodies prevents radiation-induced acceleration of metastatic cancer progression
}

\author{
Swati Biswas, ${ }^{1}$ Marta Guix,,2 Cammie Rinehart,,2 Teresa C. Dugger,2 Anna Chytil,1 \\ Harold L. Moses, ${ }^{1,3,4}$ Michael L. Freeman, ${ }^{5}$ and Carlos L. Arteaga ${ }^{1,2,4}$ \\ ${ }^{1}$ Department of Cancer Biology, ${ }^{2}$ Department of Medicine, ${ }^{3}$ Department of Pathology, ${ }^{4}$ Breast Cancer Research Program, \\ Vanderbilt-Ingram Cancer Center, and 5Department of Radiation Oncology, Vanderbilt University School of Medicine, Nashville, Tennessee, USA.
}

\begin{abstract}
We investigated whether TGF- $\beta$ induced by anticancer therapies accelerates tumor progression. Using the $M M T V / P y V m T$ transgenic model of metastatic breast cancer, we show that administration of ionizing radiation or doxorubicin caused increased circulating levels of TGF- $\beta 1$ as well as increased circulating tumor cells and lung metastases. These effects were abrogated by administration of a neutralizing pan-TGF- $\beta$ antibody. Circulating polyomavirus middle $T$ antigen-expressing tumor cells did not grow ex vivo in the presence of the TGF- $\beta$ antibody, suggesting autocrine TGF- $\beta$ is a survival signal in these cells. Radiation failed to enhance lung metastases in mice bearing tumors that lack the type II TGF- $\beta$ receptor, suggesting that the increase in metastases was due, at least in part, to a direct effect of TGF- $\beta$ on the cancer cells. These data implicate TGF- $\beta$ induced by anticancer therapy as a prometastatic signal in tumor cells and provide a rationale for the simultaneous use of these therapies in combination with TGF- $\beta$ inhibitors.
\end{abstract}

\section{Introduction}

TGF- $\beta$ is both a tumor suppressor and a tumor promoter. The TGF- $\beta$ ligands bind to cognate serine/threonine kinase transmembrane receptors, which in turn phosphorylate and activate the Smad family of signal transducers. Once activated, Smad 2 and $S m a d 3$ associate with Smad4 and translocate to the nucleus, where they regulate the transcription of genes involved in cell cycle arrest and apoptosis (1), essential for the tumor suppressor role of the TGF- $\beta$ s. Indeed, loss or attenuation of TGF- $\beta$ signaling in epithelial cells and stroma is permissive for epithelial cell transformation $(2,3)$. On the other hand, introduction of dominant-negative TGF- $\beta$ receptors into metastatic cancer cells has been shown to inhibit epithelial-to-mesenchymal transdifferentiation, motility, invasiveness, and survival, supporting the tumor promoter role in TGF- $\beta$ in fully transformed cells (reviewed in ref. 4). Most carcinomas retain TGF- $\beta$ receptors but attenuate or lose the Smad-dependent antimitogenic effect while, in some cases, gaining prometastatic abilities in response to TGF- $\beta$. In addition, excess production and/or activation of TGF- $\beta$ by cancer cells can contribute to tumor progression by paracrine mechanisms involving modulation of the tumor microenvironment $(2,5,6)$. These data have provided a rationale in favor of blockade of autocrine/paracrine TGF- $\beta$ signaling in human cancers with a therapeutic intent.

In addition to Smads, TGF- $\beta$ can stimulate several transforming signaling pathways (7). TGF- $\beta$ has previously been shown to protect transformed cells from apoptosis (8-10). One possible mechanism for this cellular response is TGF- $\beta$-induced activation of PI3K and its target, the serine-threonine kinase Akt $(11,12)$, a signaling program associated with resistance to anticancer drugs.

Nonstandard abbreviations used: $\mathrm{PyVmT}$, polyomavirus middle T antigen; T $\beta$ RII, type II TGF- $\beta$ receptor.

Conflict of interest: C.L. Arteaga receives grant support from Lilly Research Laboratories, Genentech, and Merck and is a consultant for Sunesis, GlaxoSmithKline, AstraZeneca, and Monogram Biosciences.

Citation for this article: J. Clin. Invest. 117:1305-1313 (2007). doi:10.1172/JCI30740.
Some tumors resistant to conventional anticancer chemotherapy overexpress TGF- $\beta$ s $(13,14)$, and inhibitors of TGF- $\beta$ have been shown to reverse this resistance (15). In addition, overexpression of TGF- $\beta$ ligands have been reported in most cancers, and high levels of these in tumor tissues and/or serum are associated with early metastatic recurrences and/or poor patient outcome (16-21).

In transgenic models of breast cancer, TGF- $\beta$ signaling enhances the metastatic progression of established mammary tumors induced by oncogenes such as Neu/ErbB2 or polyomavirus middle T antigen $(P y V m T)(22-24)$. Furthermore, in transgenic mice expressing the PyVmT oncogene under the control of the MMTV/LTR mammary promoter, conditional induction of active TGF- $\beta 1$ for as little as 2 weeks increases lung metastases by more than 10-fold (10). Some anticancer therapies have been shown to induce TGF- $\beta$ systemically or in situ (25-28). Therefore, we speculated that in tumors resistant to anticancer therapies or in resistant subpopulations within those tumors, treatment-induced TGF- $\beta$ would provide a survival signal to cancer cells potentially accelerating tumor progression immediately after therapy. Using the MMTV/PyVmT transgenic model of metastatic breast cancer, we show here that administration of ionizing radiation or doxorubicin caused increased circulating levels of TGF- $\beta 1$ as well as increased circulating tumor cells and lung metastases. These effects were abrogated by administration of a neutralizing pan-TGF- $\beta$ antibody. Radiation did not increase lung metastases in mice bearing tumors that lack the type II TGF- $\beta$ receptor (T $\beta$ RII). These data implicate TGF- $\beta$ induced by anticancer therapy as a prometastatic signal in tumors and thus provide a rationale for the simultaneous use of these therapies in combination with TGF- $\beta$ inhibitors.

\section{Results}

Thoracic radiation and chemotherapy increase circulating TGF- $\beta 1$. We administered 10 Gy to the thoraxes or pelvises of 8-week-old FVB virgin female mice. Blood was collected 24 hours after irradiation. We observed an approximate 2 -fold increase in plasma TGF- $\beta 1$ in irradiated mice over controls regardless of the site of radiation (tho- 
A
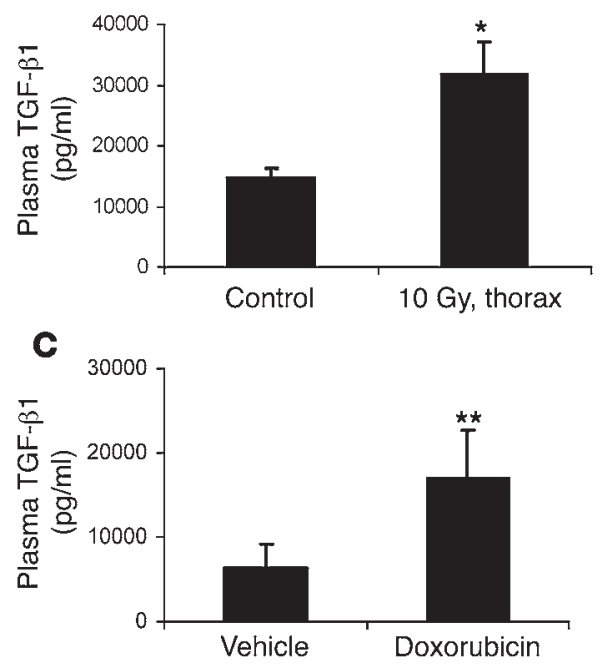

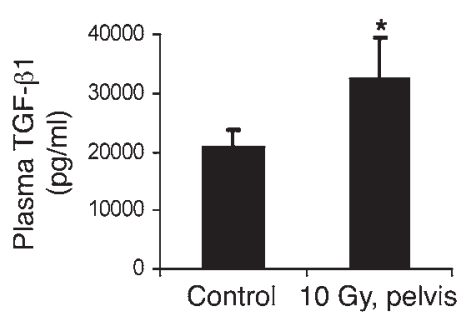

D

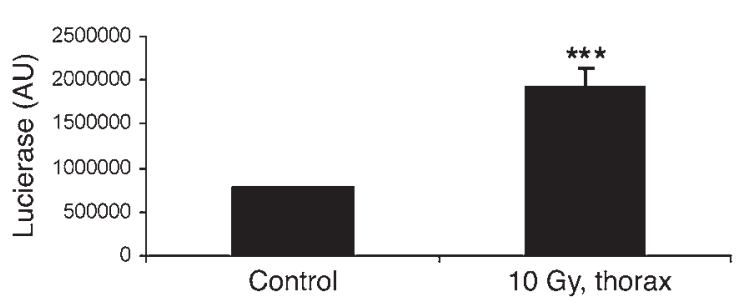

Figure 1

Radiation and chemotherapy increase circulating TGF- $\beta 1$. (A) FVB mice were subjected to 10 Gy delivered to the thorax (left) or pelvis (right). Blood was collected 24 hours later, and plasma TGF- $\beta 1$ level was measured as described in Methods. (B) Eight-week-old tumor-bearing MMTVIPyVmT mice or nontransgenic FVB mice bearing PyVmT tumors of $200 \mathrm{~mm}^{3}$ or greater in mammary fat pad no. 4 were left untreated or administered 10 Gy to the thorax. Plasma TGF- $\beta 1$ levels were measured 24 hours later. (C) Transgenic mice were treated 3 times with vehicle or doxorubicin (5 mg/kg i.p.) at 21-day intervals starting at week 8. TGF- $\beta 1$ was measured in plasma collected at week 15 . Data in A-C represent 3 independent experiments using 3 subjects per group. (D) FVB mice were administered 10 Gy to the thorax. Five weeks later, lungs from irradiated mice and controls were harvested and lysates $(250 \mu \mathrm{g} / \mathrm{ml})$ added in triplicate wells to mink lung epithelial cells that stably express a plasminogen activator inhibitor-1/luciferase reporter (PAI-1/luciferase reporter). After 24 hours, luciferase expression was measured as described in Methods. ${ }^{*} P<0.05,{ }^{* *} P<0.01,{ }^{\star * *} P<0.001$ versus control.

rax, $P=0.03$; pelvis, $P=0.02$; Figure $1 \mathrm{~A})$, while TGF- $\beta 2$ levels did not change (data not shown). Similar results were obtained in 8-weekold $M M T V / P y V m T$ transgenic mice and in nontransgenic mice transplanted with $M M T V / P y V m T$ tumor cells stably transfected with a luciferase expression vector $(P=0.015$ and $P=0.007$, respectively, versus controls; Figure $1 \mathrm{~B})$. Levels of TGF- $\beta 1$ remained higher than controls 7 days after radiation (data not shown). To expand these results to other anticancer therapies, we examined the effect of the DNA-intercalating agent and topoisomerase II inhibitor doxorubicin (Adriamycin). Transgenic mice were treated 3 times with doxorubicin ( $5 \mathrm{mg} / \mathrm{kg}$ i.p.) at 21-day intervals starting at week 8 . In plasma collected on week 15 , TGF- $\beta 1$ was also elevated 2 -fold compared with untreated mice ( $P=0.009$; Figure $1 C)$, whereas TGF- $\beta 2$ levels remained constant. To measure activated TGF- $\beta 1$ in the lung tissue harvested 5 weeks after radiation, we used a TGF- $\beta 1$ bioassay that uses mink lung epithelial cells stably expressing a plasminogen activator inhibitor-1/luciferase reporter (PAI-1/luciferase reporter) (29). Tissue lysates from irradiated mouse lungs induced a 2 -fold increase in active TGF- $\beta 1$ compared with nonirradiated lung tissue lysates $(P=0.0008$; Figure $1 \mathrm{D})$.

Thoracic radiation and chemotherapy increase circulating tumor cells and lung metastases. TGF- $\beta$ enhances the survival of tumor cells that express the PyVmT oncogene, and conditional induction of TGF- $\beta$ has previously been shown to accelerate metastases from mammary tumors arising in $M M T V / P y V m T$ transgenic mice (10). Thus, we speculated that treatment-enhanced levels of circulating TGF- $\beta 1$ may be associated with an increase in circulating tumor cells and lung metastases in this model of metastatic breast cancer. Tumorbearing MMTV/PyVmT mice were subjected to thoracic irradiation (10 Gy) at 8 weeks of age and followed until week 13. Blood was collected via heart puncture at the termination of the experiment, and its cellular fraction was evaluated for its ability to form colonies ex vivo. Blood from untreated mice produced an average of $1.6 \pm 1.5$ colonies, whereas blood from irradiated mice yielded an average of $28.3 \pm 7.6$ colonies, when measured $10-12$ days later $(P=0.02$; Figure $2 \mathrm{~A})$. Immunostaining using a $\mathrm{PyVmT}$ antibody confirmed oncogene expression in the growing colonies (Figure 2A). Consistent with the increase in viable circulating tumor cells, we observed a 17-fold increase in surface lung metastases in irradiated mice compared with untreated controls $(P=0.009$; Figure $2 B)$. Similar increases were observed in lung metastases of mice treated with doxorubicin compared with untreated mice $(P=0.032$; Figure $2 \mathrm{C})$ as well as in colony number of blood obtained 24 hours after the first dose of doxorubicin compared with that of untreated controls $(17.2 \pm 3.7$ versus $7.2 \pm 2$ colonies; $n=5 ; P=0.007)$. Tumor burden, as estimated by mouse weight and serial tumor diameters, was not different between treated and untreated mice, suggesting that the difference in TGF- $\beta 1$ levels cannot be explained simply by an increase in tumor burden over time.

We next examined if the radiation-induced increase in lung metastases was limited to tumor-bearing MMTV/PyVmT transgenic mice. To test this possibility, we injected nontransgenic syngeneic FVB mice with luciferase-expressing tumor cells derived from a transgenic PyVmT-expressing mammary cancer. In these mice, tumor 
A

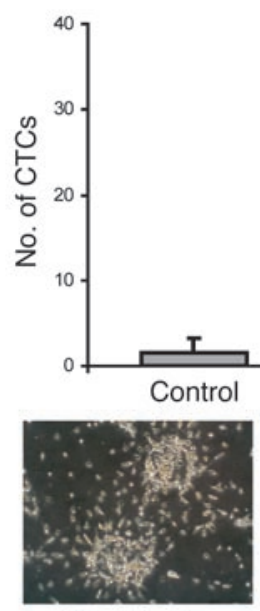

Brightfield
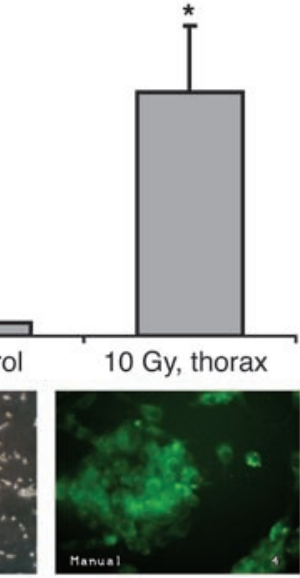

PyVmT

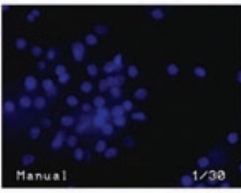

Hoechst

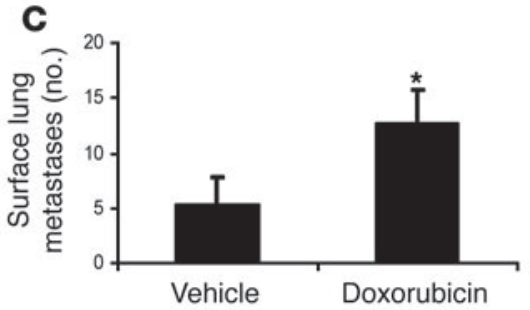

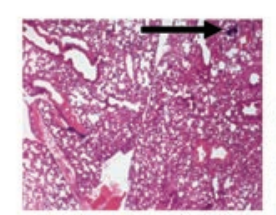

Vehicle
B

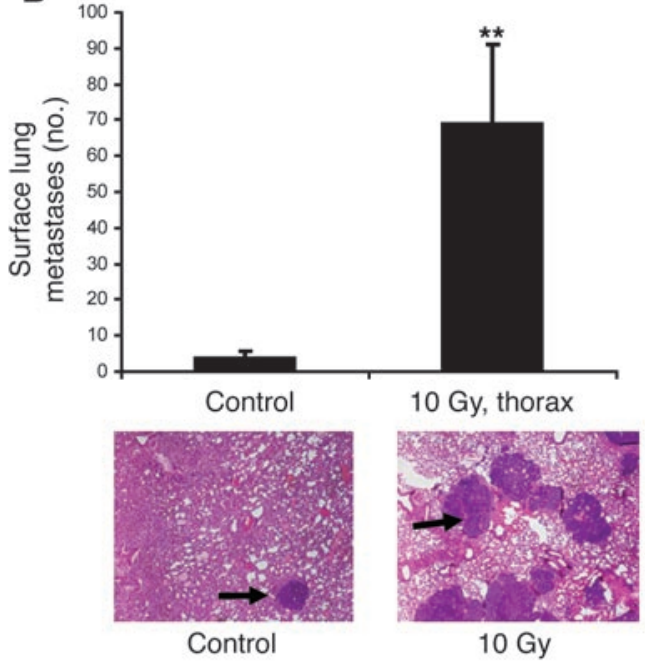

Figure 2

Radiation and chemotherapy increase circulating tumor cells and lung metastases. (A) Female MMTV/PyVmT female mice were subjected to thoracic irradiation at 8 weeks of age. At 13 weeks, blood was collected via heart puncture at the termination of the experiment, and its cellular fraction evaluated for its ability to form colonies ex vivo as described in Methods. CTCs, circulating tumor cells. Representative images of colonies arising from single circulating tumor cells harvested from mouse blood are shown below. Transgene-positive colonies were assessed under a fluorescence microscope using a PyVmT antibody and a fluorescent secondary antibody. (B) In same mice as in $\mathbf{A}$, surface lung metastases were counted at 13 weeks of age. Data in $\mathbf{A}$ and $\mathbf{B}$ are representative of 3 independent experiments with 4 mice per group. Representative H\&Estained sections of lung tissues from control and irradiated transgenic mice obtained 5 weeks after thoracic radiation are shown below. Black arrows indicate lung metastases. (C) Eight-week-old tumor-bearing MMTV/PyVmT transgenic mice were treated 3 times with vehicle or doxorubicin (5 mg/kg i.p.) at 21-day intervals. The experiment was terminated on week 15, and surface lung metastases were counted. Representative $\mathrm{H} \& \mathrm{E}$-stained lung sections containing metastatic foci are shown at right. Original magnification, $\times 100$. ${ }^{*} P<0.05$, ${ }^{* \star} P<0.01$ versus control.

growth in the left inguinal (no. 4) mammary fat pad and metastatic dissemination to the lungs was estimated by monitoring bioluminescence (Figure 3A). Once tumors reached a volume of $200 \mathrm{~mm}^{3}$ or greater, mice were administered $10 \mathrm{~Gy}$ to the thorax while shielding the rest of the body and sacrificed 2 weeks later. There was a 3 -fold increase in surface lung metastases in irradiated mice compared with controls $(P=0.005$; Figure $3, \mathrm{~B}$ and $\mathrm{C})$. Furthermore, at 24 hours and 2 weeks after radiation, there were 8 - and 5 -fold increases, respectively, in circulating tumor cells of treated mice compared with untreated mice (Table 1). Primary tumor growth was not different between both groups (data not shown).

Exposure of PyVmT cells in culture to 1.25-7.50 Gy resulted in an increase in TGF- $\beta$ production (Figure 4A). In the studies described above, mice with established tumors were irradiated; therefore, we sought to rule out that the increase in circulating TGF- $\beta$ was the result of an effect of radiation on the cancer cells. We administered $10 \mathrm{~Gy}$ to the thoraxes of tumor-free virgin female FVB mice. One hour after irradiation, PyVmT-expressing tumor cells stably transfected with luciferase were injected via the tail vein. Tumor cell burden in the lungs was monitored by bioluminescence both in vivo and ex vivo after tumor cell injection. Lungs from irradiated mice exhibited a higher bioluminescent signal than did untreated controls (Figure 4B), which correlated with the manually counted number of surface lung metastases and with lung weight (Figure 4, C and D).

TGF- $\beta$-neutralizing antibody blocks radiation-induced increase in lung metastases. To determine whether the radiation-induced increase in circulating TGF- $\beta$ played a causal role in the increase in lung metastases, we used the $2 \mathrm{G} 7$ neutralizing pan-TGF- $\beta$ IgG2 . This monoclonal antibody blocks all 3 TGF- $\beta$ mammalian isoforms and has shown efficacy in vivo (30-32). Eight-week-old MMTV/PyVmT transgenic mice were treated with $15 \mathrm{mg} / \mathrm{kg} 2 \mathrm{G} 7$ or PBS i.p. 2 hours prior to thoracic radiation. Treatments were continued twice a week until week 13, when mice were sacrificed and evaluated for the presence of lung metastases. Treatment with 2 G7 prevented the radiation-induced increase in lung metastases. Interestingly, however, administration of $2 \mathrm{G} 7$ to mice that were not irradiated resulted in a minor increase in lung metastases, but this difference did not reach statistical significance (Figure 5, A and B). There was no difference in primary tumor burden between both groups (data not shown).

The number of circulating tumor cells was estimated by collecting blood and culturing the cellular fraction ex vivo. The number of growing colonies was markedly reduced in blood from irradiated 
A

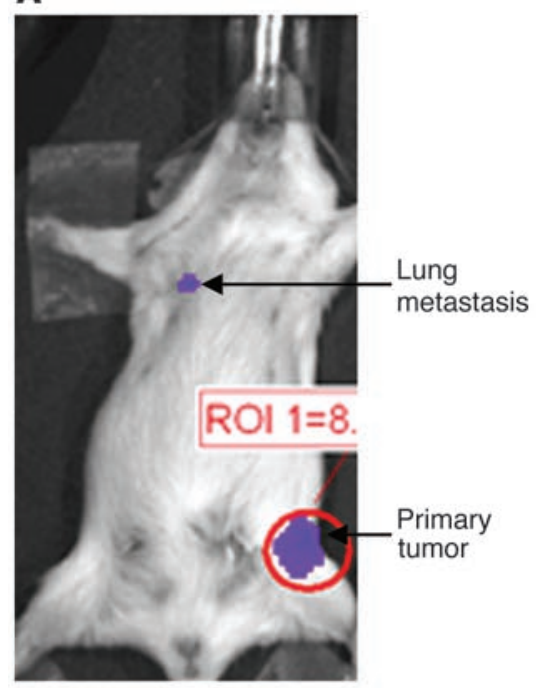

B

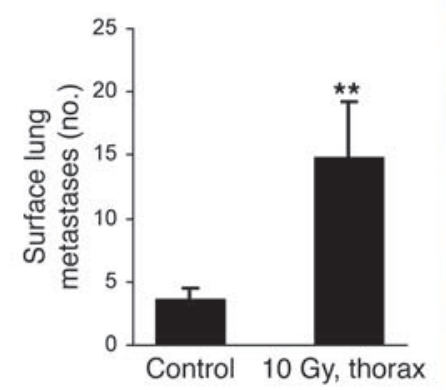

C

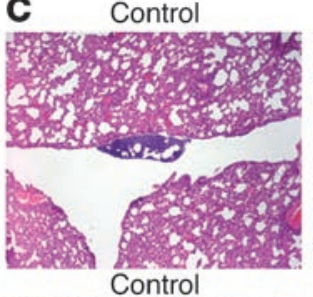

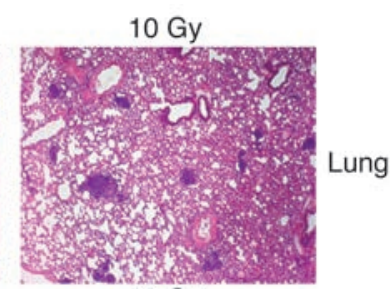

10 Gy
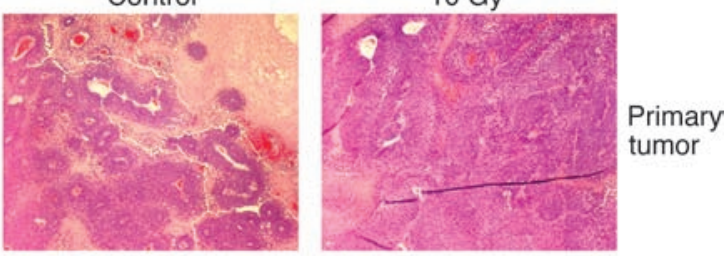

\section{Figure 3}

Thoracic radiation increases lung metastases from tumor transplants. (A) MMTV/PyVmT cells stably expressing luciferase were injected in the mammary fat pads of syngeneic FVB mice. Bioluminescence imaging was used to monitor tumor growth and lung metastases twice a week thereafter. A representative mouse imaged 2 weeks after cell inoculation is shown. (B and C) Mice with PyVmT/Luc tumors measuring at least 200 mm ${ }^{3}$ were treated or not with thoracic irradiation (10 Gy). (B) Surface lung metastases were quantitated 2 weeks later. Data are mean \pm SD of 5 mice per group in 1 of 2 experiments. ${ }^{\star *} P<0.01$ versus control. (C) H\&E slides of lung and primary tumor sections. Original magnification, $\times 100$.

and nonirradiated mice that had been treated with 2G7 (Figure 5C). Finally, we examined whether inhibition of TGF- $\beta$ by the addition of 2G7 ex vivo would prevent outgrowth of tumor cell colonies from mouse blood. Blood from tumor-bearing irradiated mice was seeded in the presence or absence of $2 \mathrm{G} 7$, and colony growth was assessed 10 days later. Incubation with $2 \mathrm{G} 7$ reduced the number of colonies by $70 \%$ (Figure 5D), suggesting that autocrine TGF- $\beta$ is a survival factor for PyVmT-expressing circulating cancer cells.

TGF- $\beta$ receptors in tumor cells are required for the radiation-induced increase in lung metastases. The results described above suggest that the increase in circulating TGF- $\beta$ induced by radiation serves as a survival signal for circulating tumor cells, which are then capable of colonizing and growing in the lung. We thus reasoned that the absence of T $\beta R I I$ in MMTV/PyVmT tumors, achieved by eliminating the cellular response to circulating TGF- $\beta$, would abrogate this effect of radiation on metastases. To examine this possibility, we used PyVmT-expressing tumor cells in which T $\beta$ RII had been conditionally deleted using Cre-Lox technology (33). PyVmT-expressing tumor cell lines with or without TGFBR2 (referred to hereafter as PyVmT/TGFBR2 flox/flox or PyVmT/TGFBR2 $2^{K O}$ ) were generated from mammary cancers in MMTV/PyVmT/TGFBR2 flox/flox and MMTV/ PyVmT/TGFBR2 ${ }^{K O}$ mice, respectively. PyVmT/TGFBR2flox/flox and PyVmT/TGFBR2 ${ }^{K O}$ cell lines were stably transfected with a vector encoding for luciferase and injected via the tail vein in 8-week-old virgin female FVB mice that were nonirradiated or administered 10 Gy to the thoracic cavity 1 hour prior to tumor cell injection. Lung metastases were assessed 2 weeks later. In mice injected with PyVmT/TGFBR2flox/flox cells, there was both an increase in bioluminescence and a 6-fold increase in lung metastases in irradiated versus untreated mice, whereas there was no difference between irradiated and nonirradiated mice injected with cells lacking T $\beta R I I$ (Figure 6, A-C). The PyVmT/TGFBR2 ${ }^{K O}$ tumor nodules were larger than the PyVmT/ TGFBR2flox/flox nodules. This is consistent with a previous report showing that loss of T $\beta \mathrm{RII}$ in PyVmT-expressing mammary cancer cells increased growth of pulmonary metastases (33). PCR-amplified DNA from tumor tissue confirmed the recombination in PyVmT/TGFBR2 ${ }^{K O}$ cells (Figure 6D).

\section{Discussion}

We investigated whether TGF- $\beta$ induced by anticancer therapies would accelerate tumor progression in a transgenic model of metastatic breast cancer. In $M M T V / P y V m T$ transgenic mice, administration of ionizing radiation to the lungs or treatment with doxorubicin increased circulating levels of TGF- $\beta 1$ as well as circulating tumor cells and lung metastases. The rise in circulating TGF- $\beta 1$ levels and subsequent increase in metastases did not require the presence of tumors in mice at the time of treatment, as they were also seen in tumor-free mice inoculated with tumor cells after radiation. Induction of TGF- $\beta 1$ was not specific to radiation of the thorax, because it

\section{Table 1}

Lung radiation contributes to circulating tumor cells' survival

$\begin{array}{lcc}\text { Time after RT } & \text { Dose } & \text { Circulating tumor cells } \\ 24 \text { hours } & 0 \mathrm{~Gy} & 2 \pm 2 \\ 24 \text { hours } & 10 \mathrm{~Gy} & 17.3 \pm 6.2^{\mathrm{A}} \\ 2 \text { weeks } & 0 \mathrm{~Gy} & 4.5 \pm 0.7 \\ 2 \text { weeks } & 10 \mathrm{~Gy} & 50 \pm 15^{\mathrm{A}}\end{array}$

MMTV/PyVmT cells stably expressing luciferase were injected in the right inguinal mammary fat pads of FVB mice. Mice with tumors measuring at least $200 \mathrm{~mm}^{3}$ were nonirradiated or administered 10 Gy to the thorax. Blood was collected by heart puncture 24 hours and 2 weeks after radiation, and its cellular fraction was evaluated for its ability to form colonies ex vivo as described in Methods. Data are mean \pm SD of 5 mice per group and represent 2 separate experiments. $R T$, radiation therapy. ${ }^{A} P=0.016$ versus 0 Gy at the same time point. 

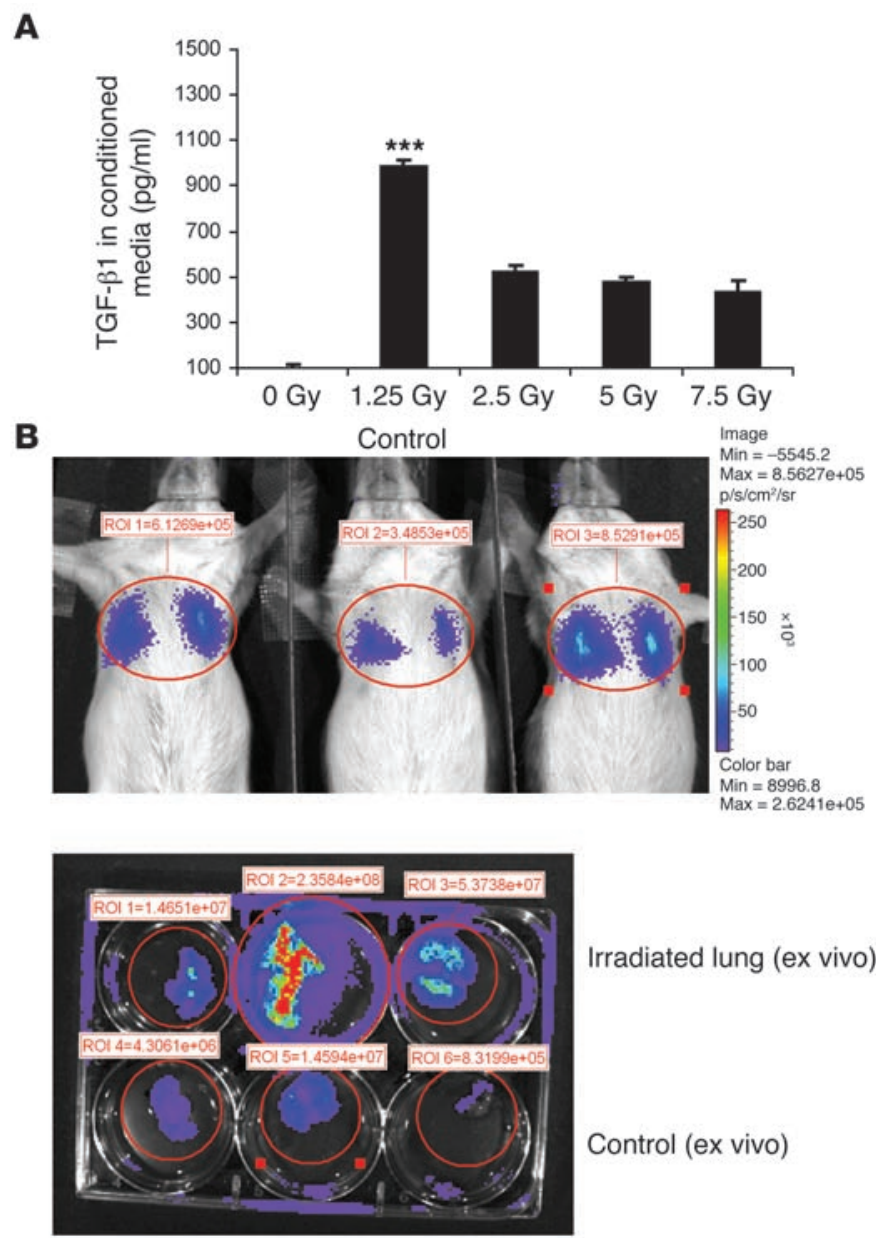

Irradiated lung (ex vivo)
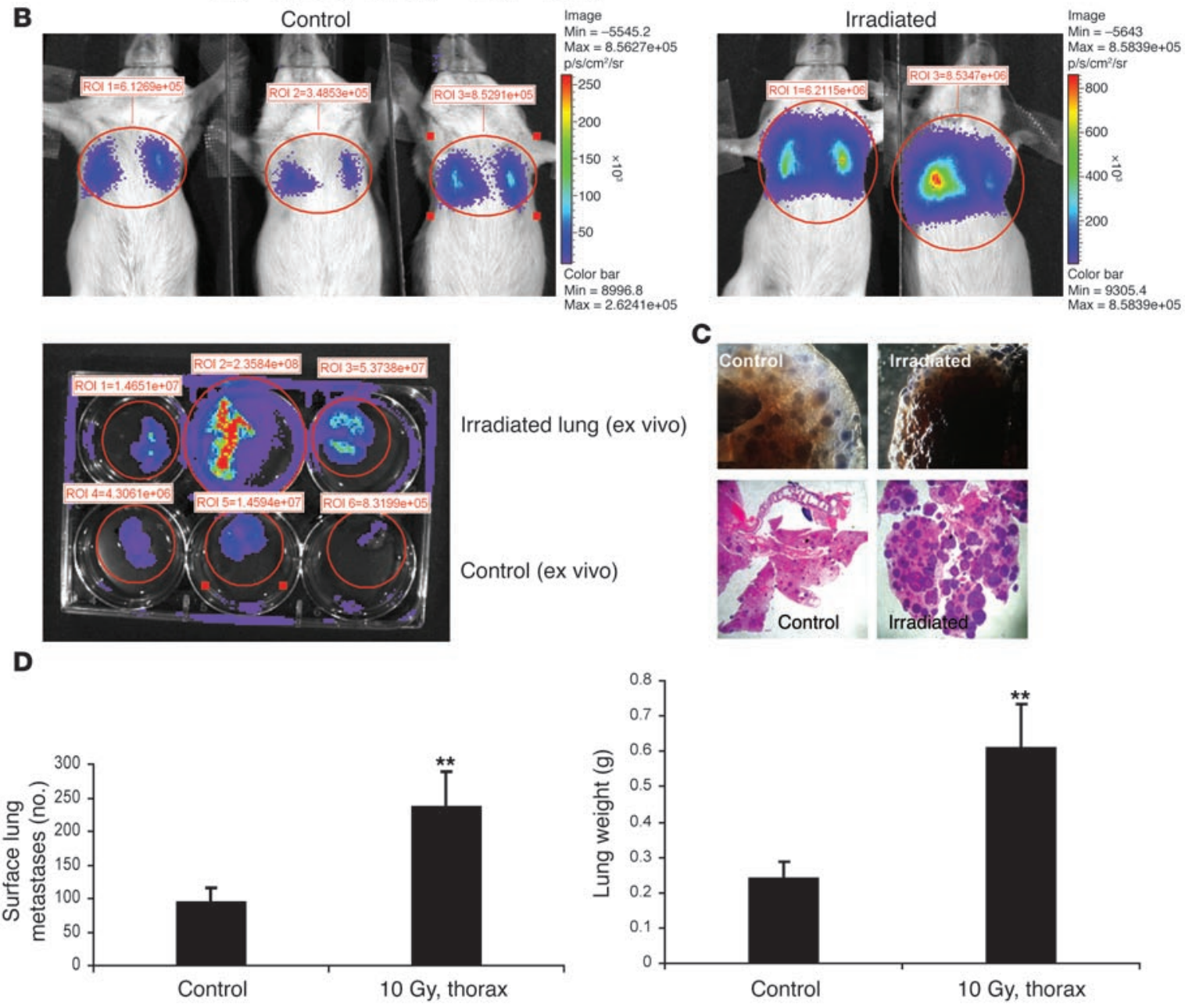

Figure 4

Prior radiation is permissive for metastatic lung colonization in tumor-free mice. (A) MMTVIPyVmT cells in 100-mm dishes in serum-free medium were treated with 1.25-7.5 Gy. Cell-conditioned medium was collected 72 hours later, and TGF- $\beta 1$ levels were determined by ELISA as described in Methods. (B) MMTVIPyVmT cells stably expressing luciferase were injected via tail vein in virgin female FVB mice. Where indicated, mice received $10 \mathrm{~Gy}$ to the thorax 1 hour prior to injection of cells. Cancer cells in lungs were visualized by mouse bioluminescence 2 weeks after inoculation (top). In some cases, lungs were surgically removed after administration of D-luciferin and imaged ex vivo (bottom). Controls are shown on the left and irradiated mice are shown on the right. (C) Representative lung whole mounts (top) and H\&E sections of lungs (bottom; original magnification, $\times 100$ ) from control and irradiated mice. (D) Quantification of surface lung metastasis (left) and lung weight (right) in control and irradiated mice. Data are mean \pm SD of 5 mice per group in 2 independent experiments. ${ }^{\star *} P<0.01$, ${ }^{\star * \star} P<0.001$ versus control.

was also observed after pelvic radiation and after systemic treatment with doxorubicin. The increase in both circulating tumor cells and lung metastases following radiation was abrogated by administration of 2G7. Importantly, circulating PyVmT-expressing tumor cells failed to grow ex vivo in the presence of $2 \mathrm{G} 7$ (Figure 5D), which sug- gests that autocrine TGF- $\beta$ is a survival signal in these cells. These results are consistent with 2 previous studies in which blockade of TGF- $\beta$ with a soluble T $\beta$ RII:Fc fusion protein or stable transfection with antisense TGF- $\beta 1$ inhibited tumor cell motility, survival and intravasation as well as lung metastases $(10,23)$. 
A

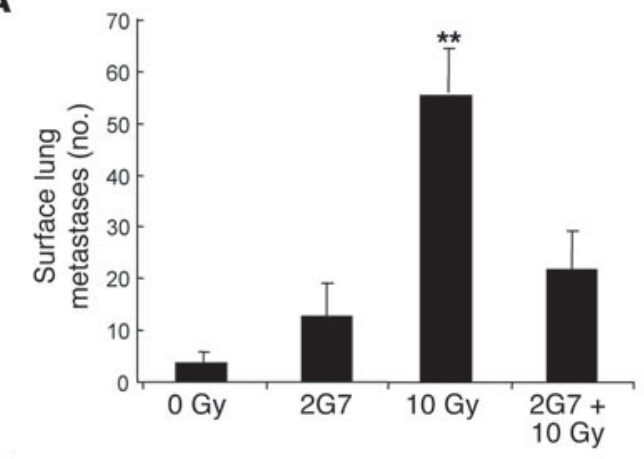

B

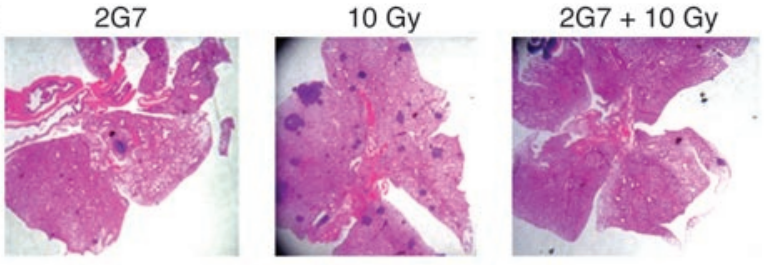

C
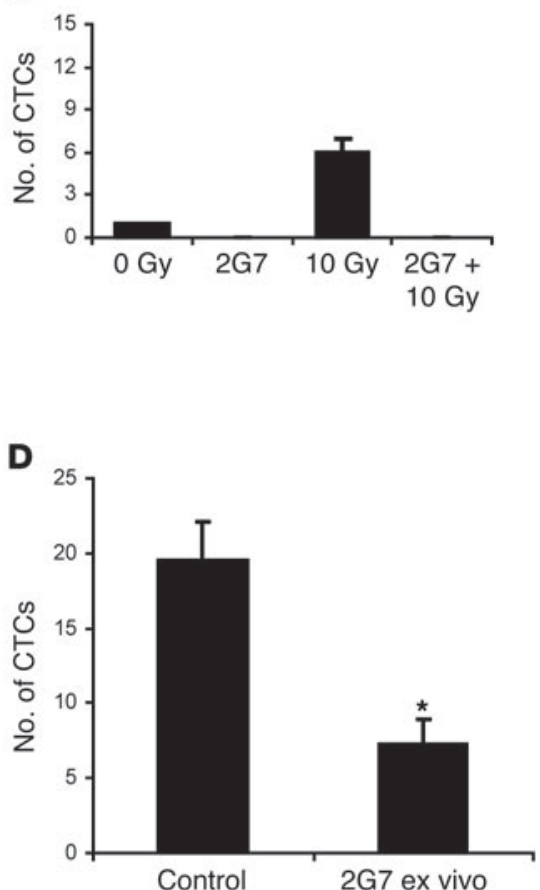

\section{Figure 5}

TGF- $\beta$-neutralizing antibody 2 G7 blocks radiation-induced increase in lung metastases. (A and B) Eight-week-old tumor-bearing MMTV/PyVmT mice were administered $10 \mathrm{~Gy}$ to the thorax. Where indicated, mice were treated with $15 \mathrm{mg} / \mathrm{kg}$ of $2 \mathrm{G} 7$ twice a week until week 13 , at which time surface lung metastases were counted $(\mathbf{A})$. Data are mean \pm SD of 5 mice per group. (B) Representative H\&E stains of lung sections. The experiment was repeated once with similar results. (C) Blood was collected at the completion of the experiment via heart puncture and its cellular fraction evaluated for its ability to form colonies ex vivo as described in Methods. (D) At 13 weeks, blood was collected from tumor-bearing transgenic mice that were exposed to thorax irradiation. The cellular fraction was plated ex vivo as in $\mathbf{C}$ in the presence of $20 \mu \mathrm{g} / \mathrm{ml} 2 \mathrm{G} 7$ or PBS. Colonies measuring $50 \mu \mathrm{m}$ or greater were counted manually $10-12$ days later. Data are mean \pm SD of 5 mice per group. ${ }^{*} P<0.05,{ }^{* \star} P<0.01$ versus control.

Radiation failed to enhance lung metastases in mice bearing tumors that lack T $\beta$ RII. To demonstrate this, we used cell lines from established mammary tumors in MMTV/PyVmT/TGFBR2 flox/flox and $M M T V / P y V m T / T G F B R 2^{K O}$ mice. Forrester et al. have reported that upon conditional deletion of TGFBR2 by expression of MMTV/Cre, the knockout PyVmT-expressing tumors develop after a much shorter latency and exhibit markedly increased pulmonary metastases compared with TGFBR2flox/flox tumors (33). Since we used i.v. injected cell lines derived from established tumors with or without T $\beta$ RII, our results do not address the difference in latency and metastatic behavior reported by Forrester et al. (33). Although we cannot rule out an effect of increased TGF- $\beta$ on the host microenvironment and/or immune system in irradiated mice that in turn facilitated metastatic progression, the result with tumors lacking $\mathrm{T} \beta \mathrm{RII}$ strongly suggests that the increase in metastases was at least in part due to a direct effect of TGF- $\beta$ on the cancer cells.

The signaling responses in tumor cells potentially modulated by circulating TGF- $\beta$ are unclear at this point. However, the prometastatic effect of TGF- $\beta$ is not limited to this transgenic model. For example, bigenic mice expressing active TGF- $\beta 1$ and Neu under the control of the MMTV/LTR promoter also exhibit more circulating tumor cells and lung metastases than do MMTV/Neu mice. Invasion into Matrigel and motility through transwells of mammary tumor cells expressing both Neu and active TGF- $\beta 1$ is blocked by soluble T $\beta$ RII:Fc, suggesting that Neu alone is not sufficient for inducing an invasive phenotype (24). Furthermore, in transgenic tumors expressing activated Neu, coexpression of an active mutant of Alk5, T $\beta R$ I, increases frequency of extravascular lung metastases (22), consistent with the effect of TGF- $\beta$ on peritumoral proteases and cancer cell adhesion and invasion. The potent antimetastatic effect of 2G7 against tumor cells expressing a potent oncogene such as PyVmT, known to activate Erk and PI3K, is somewhat counterintuitive. It suggests, however, that TGF- $\beta$ can amplify oncogene signals above a threshold required for a fully efficient metastatic phenotype, and conversely, that blockade of TGF- $\beta$ signaling in oncogene-transformed cells would reduce those signals below the threshold required for oncogene-induced progression. Indeed, forced expression of dominant-active $\operatorname{Smad} 2$ in squamous cancers has been shown to cooperate with active Ras on the conversion of noninvasive to metastatic tumors (34), while expression of Smad mutants that do not bind T $\beta$ RI suppresses breast cancer cell metastases (35). Finally, expression of dominant-negative truncated T $\beta \mathrm{RI}$ in Ras-transformed cells inhibits tumorigenicity and metastases (36).

The repopulation and progression of tumors after anticancer therapy is a well-recognized phenomenon. It has been shown to occur following radiotherapy, chemotherapy, and surgery (reviewed in ref. 37). Increased TGF- $\beta$ in response to radiation has also been linked to lung injury after radiation as a result of increased collagen deposition and alveolar wall thickness as well as endothelial damage (28). Radiation-induced lung tissue damage is markedly reduced by administration of an anti-TGF- $\beta$ antibody (38). In the case of surgery, it has been proposed that excessive release of 
A

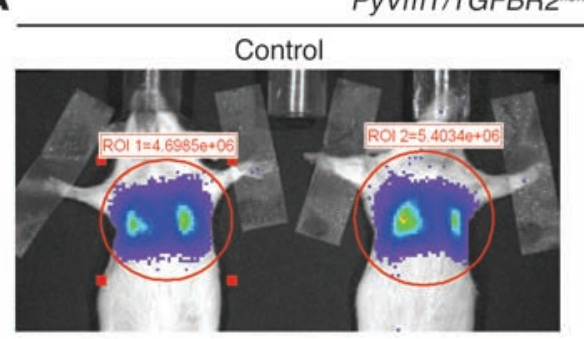

B
PyVmT/TGFBR2 $2^{\text {10xthox }}$
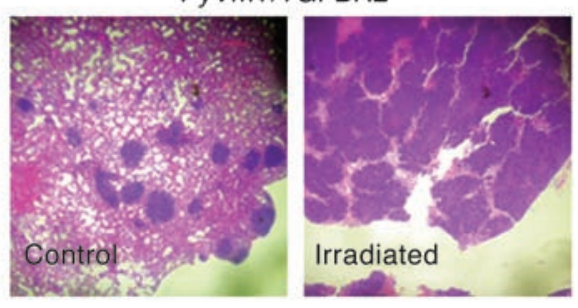

C

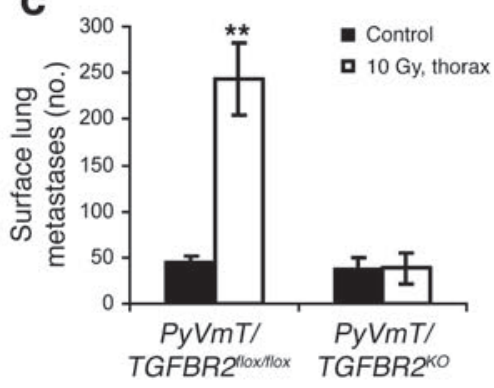

PyVmT/TGFBR2 ${ }^{\text {KO }}$
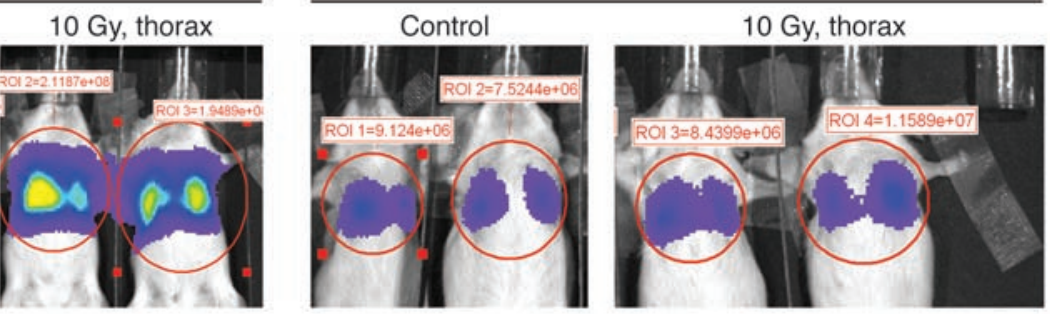

PyVmT/TGFBR2Ko
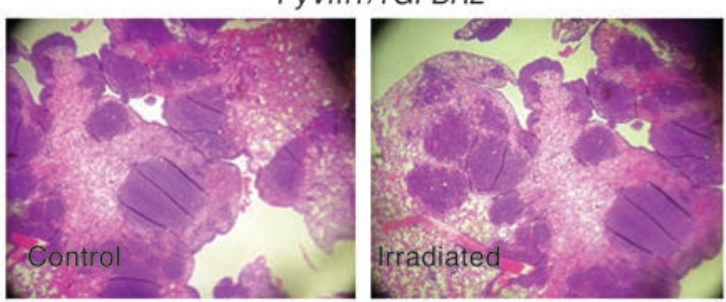

D

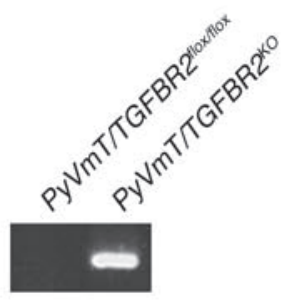

Figure 6

Absence of TGF $\beta$ RII in tumor cells abrogates radiation-induced increase in lung metastases. (A-C) PyVmT/TGFBR2flox/flox and PyVmT/TGFBR2KO cells stably expressing luciferase were injected via the tail vein in virgin 8-week-old female FVB mice that had received or not $10 \mathrm{~Gy}$ to the thorax 1 hour prior to tumor cell injection. Two weeks later, surface lung metastases were evaluated by bioluminescence (A), by histology (B), and by manually counting surface lung metastases (C) as described in Methods. Data are mean \pm SD of 4 mice per group. (D) PCR from genomic DNA extracted from both cell lines, showing the presence of a recombined band only in PyVmT/TGFBR2 ${ }^{K O}$ cells. ${ }^{\star \star} P<0.01$ versus control.

growth factors, including TGF- $\beta$, as a result of tissue manipulation and wound healing promotes metastases in the immediate postoperative period (39). Indeed, tumor cells are found in the circulation and their numbers increase immediately after surgery (40), and persistence of high circulating TGF- $\beta$ levels 2 weeks after curative resection of colorectal cancers predicts early metastatic recurrences in the liver (20). Conversely, serum levels of TGF- $\beta$ decrease significantly after curative surgical resection of colorectal tumors (41). Finally, in advanced head and neck cancers treated with chemoradiotherapy and in non-small-cell lung cancers treated with chemotherapy, a decrease in circulating TGF- $\beta$ levels has been shown to correlate with response to treatment $(42,43)$.

In this report, we show that radiation and chemotherapy increased circulating levels of TGF- $\beta$, circulating cancer cells, and tumor metastases. These effects were blocked by systemic administration of $2 \mathrm{G} 7$, thus supporting a causative role for TGF- $\beta$ in the metastatic progression observed after therapy. These data have several clinical implications. First, increased circulating TGF- $\beta$ in response to cancer therapy should be prospectively investigated and monitored, as it may represent a marker of tumors destined to progress rapidly after therapy. Second, patients in whom increased circulating TGF- $\beta$ is observed may bear cancers in which administration of a TGF- $\beta$ inhibitor can add to the effect of the primary therapy as well as abrogate therapy-related toxic effects such as radiation-induced tissue damage and fibrosis. With currently available methods and therapeutic TGF- $\beta$ inhibitors in early clinical development, these hypotheses can be prospectively examined in the near future.

\section{Methods}

Reagents and cell lines. The 2G7 hybridoma (30) was a gift from B. Fendly (Genentech). It was generated and affinity purified at the Vanderbilt University Molecular Recognition Core facility. Recombinant human TGF- $\beta 1$ and the Quantikine Elisa kit for TGF- $\beta 1$ were from R\&D Systems. Cell lines expressing PyVmT were derived from mammary tumors arising in MMTV/PyVmT

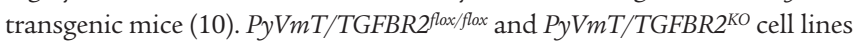
were generated from mammary cancers in MMTV/PyVmT/TGFBR2flox/flox and $M M T V / P y V m T / T G F B R 2^{K O}$ mice (33), respectively. T $\beta R I I$ had been conditionally deleted in the mammary gland of these mice using Cre/Lox technology. Cells were maintained in a humidified $5 \% \mathrm{CO}_{2}$ incubator at $37^{\circ} \mathrm{C}$ and in DMEM (Cellgro; Mediatech Inc.) supplemented with 10\% FBS (HyClone).

TGF- $\beta 1$ bioassay. Mink lung epithelial reporter cells stably expressing firefly luciferase under the control of the PAI-1 promoter (29) were seeded in 12 -well plates $\left(2 \times 10^{5} /\right.$ well $)$ and allowed to adhere overnight. A known concentration of soluble TGF- $\beta 1$ or lung tissue lysates $(250 \mu \mathrm{g} /$ well) was added in triplicate, and the cells were incubated for an additional 24 hours in $5 \% \mathrm{CO}_{2}$ at $37^{\circ} \mathrm{C}$. After washes, the cells were harvested in $200 \mu \mathrm{l} /$ well 
of lysis buffer (Dual Luciferase Kit; Promega), and luciferase activity was measured following the manufacturer's protocol.

DNA extraction and PCR. Paraffin-embedded tumor sections were deparaffinized and rehydrated; while still wet, tumor areas were scraped with a sterile razor blade and collected in a sterile eppendorf tube. DNA was extracted using Instagene (Bio-Rad) following the manufacturer's protocol. DNA samples were stored at $-20^{\circ} \mathrm{C}$ until further use. Cre-induced recombination in PyVmT/TGFBR2 $2^{K O}$ tumors was confirmed using PCR primers and reaction conditions described previously (44).

Retroorbital blood collection and quantification of TGF- $\beta 1$. Mice were anesthetized with $1 \%-2 \%$ isofluorane. Blood (about $250 \mu \mathrm{l} /$ mouse) was collected from the conjunctival vein in the eye using a heparinized Natelson tube (Fisher Scientific) and then transferred to heparinized glass tubes. Plasma was prepared using Ficoll-Paque Plus (Amersham Biosciences) according to the manufacturer's instructions with an additional step of centrifugation at $10,000 \mathrm{~g}$ for 10 minutes at $4^{\circ} \mathrm{C}$ for removal of platelets. For measuring TGF- $\beta 1$ in medium conditioned by PyVmT-expressing cells, $1 \times 10^{6}$ cells were plated in $100-\mathrm{mm}$ dishes in complete medium and allowed to adhere. The next day, the medium was changed to serum-free medium. After incubation overnight, cells were subjected to 1.25-7.5 Gy. After 72 hours, the medium was collected and concentrated ( $3 \mathrm{ml}$ to $500 \mu \mathrm{l})$ using speed vacuum. Both mouse plasma and cell-conditioned medium were next tested in a TGF- $\beta 1$ Quantikine Elisa kit (R\&D Systems) following acid activation as indicated in the manufacturer's protocol. A standard curve using $31.5-2,000 \mathrm{pg} / \mathrm{ml}$ human recombinant TGF- $\beta 1$ was generated and used to calculate the TGF- $\beta$ equivalents in mouse plasma or cell medium. Each specimen was examined in duplicate for a total of 3 times.

Quantification of TGF- $\beta 2$. Plasma samples were tested using TGF- $\beta 2$ Quantikine Elisa kit (R\&D Systems) following acid activation as indicated in the manufacturer's protocol. A standard curve using $31.5-2,000 \mathrm{pg} / \mathrm{ml}$ human recombinant TGF- $\beta 2$ was generated using the kit reagents and used to calculate the TGF- $\beta 2$ equivalents in mouse plasma. Each specimen was examined in duplicate for a total of 3 times.

Radiation treatment of mice. Anesthetized MMTV/PyVmT transgenic mice or normal FVB female mice (Harlan) were secured on their backs on a stage. A single anterior thoracic field was employed, with lead blocks shielding the remainder of the body. In some experiments, a single anterior abdominal field was employed. Mice were irradiated with $300 \mathrm{kVp}$ x-rays at a dose of $2.05 \mathrm{~Gy} / \mathrm{min}$. All mice were maintained in a specific pathogen-free facility in accordance with the Institutional Animal Care and Use Committee of Vanderbilt University Medical Center. Animals were fed regular diet and observed closely for any signs of distress following radiation exposure or other treatments.

Doxorubicin treatment. 53-day-old MMTV/PyVmT mice treated i.p. 3 times with doxorubicin (Adriamycin; $5 \mathrm{mg} / \mathrm{kg}$; Sigma-Aldrich) dissolved in saline at 21-day intervals until 95 days. Blood samples and lung tissue were collected from vehicle- and doxorubicin-treated mice at day 107 upon necropsy. For checking circulating tumor cells after doxorubicin treatment, a single dose $(5 \mathrm{mg} / \mathrm{kg})$ of treatment was followed, and blood was collected after 24 hours from either doxorubicin- or vehicle-treated mice for culturing circulating tumor cells.

Detection of circulating tumor cells. Circulating tumor cells were cultured following the method described previously (45), with slight modifications. Briefly, blood was collected via heart puncture. The cellular fraction (including the buffy coat and red blood cells) was separated from plasma and seeded on 6-well plates coated with growth factor-reduced Matrigel (BD Biosciences) supplemented with DMEM plus $10 \% \mathrm{FBS}$ followed by incubation at $37^{\circ} \mathrm{C}$ in a $5 \% \mathrm{CO}_{2}$ incubator. The next day each well was gently washed several times with red blood cell removal buffer $\left(4.15 \mathrm{~g} \mathrm{NH}_{4} \mathrm{Cl}, 0.5 \mathrm{~g} \mathrm{NaHCO}_{3}, 0.0186 \mathrm{~g}\right.$ disodium EDTA in $200 \mathrm{ml}$ water) and PBS. DMEM plus $10 \% \mathrm{FBS}$ ( $3 \mathrm{ml} /$ well) was added, and fresh medium was replenished every 3 days. After 10-12 days, colonies measuring $50 \mu \mathrm{m}$ or greater were counted manually. PyVmT expression was detected by immunocytochemistry. In brief, medium was aspirated and wells were washed with PBS. The colonies were fixed with $10 \%$ neutralbuffered formalin for 30 minutes at room temperature followed by 2 washes with PBS and incubation in 3\% BSA in PBS for 1 hour at room temperature. Plates were next incubated overnight at $4^{\circ} \mathrm{C}$ with a biotin-labeled mouse monoclonal antibody against PyVmT (diluted 1:500, BIOT-115L; Covance). A streptavidin-conjugated fluorescent secondary antibody (Oregon Green 488; Invitrogen) was then added for 1 hour in room temperature followed by Hoechst nuclear staining ( $1 \mu \mathrm{g} / \mathrm{ml}$ for 10 minutes). Immunofluorescence was monitored using a Leica DM IRB inverted microscope.

Generation of retroviral vectors and cell transduction. MMTV/PyVmT cells were stably transfected with a vector encoding luciferase. A luciferase cassette was excised from pGL3-Basic (Promega) and inserted into the multiple cloning site of pMSCV-puro (Clontech). Transfection of amphotropic packaging Phoenix cells was performed to generate infectious virions as described previously (46). Subconfluent cultures of oncogene-expressing cells were transduced with viral supernatant for 6 hours in the presence of $4 \mu \mathrm{g} / \mathrm{ml}$ Polybrene (Sigma-Aldrich). Medium containing viral particles was changed after 6 hours, and DMEM supplemented with 10\% heat-inactivated FCS was added. Puromycin (10 $\mu \mathrm{g} / \mathrm{ml}$; Sigma-Aldrich) was added 48 hours later, and drug-resistant colonies were pooled after 7-10 days.

Tumorigenicity and metastases studies. MMTV/PyVmT/Luc cells $\left(2 \times 10^{6}\right.$ cells in $200 \mu \mathrm{l}$ PBS) were injected in the left no. 4 mammary fat pad of FVB female mice under 1\%-2\% isofluorane anesthesia. Briefly, the fat pad was surgically exposed prior to tumor cell injection and the wound was closed using sterile clips, which were removed 10 days later. Tumors were monitored twice a week by bioluminescence and measured serially with calipers; their volume in $\mathrm{mm}^{3}$ was calculated by the formula $v=\left(w^{2} \times l\right) / 2$, where $v, w$, and $l$ represent volume, width, and length, respectively. Once tumors reached a volume of $200 \mathrm{~mm}^{3}$ or greater (in approximately 2 weeks), mice were left untreated or subjected to thoracic radiation. Mice continued to be monitored by bioluminescence twice a week until the end of the experiment. Two weeks later, mice were sacrificed and primary tumors and lungs were harvested. Surface lung metastases were counted under a dissecting microscope and the lungs were subsequently fixed in $10 \%$ neutral-buffered formalin. Fixed tumor sections were stained with $\mathrm{H} \& \mathrm{E}$ and examined microscopically for the presence of lung metastases. Blood was collected via heart puncture to measure plasma TGF- $\beta$ and circulating tumor cells as described above.

In other cases, we used $M M T V / P y V m T, P y V m T / T G F B R 2^{\text {flox } / \text { flox }}$, or $P y V m T / T G F B R 2^{K O}$ cells expressing luciferase. A single-cell suspension of $2.5 \times 10^{6}$ was prepared and reconstituted in sterile PBS; $0.5 \times 10^{6}$ cells (in $200 \mu \mathrm{l})$ were injected in the lateral tail veins of FVB female mice. Localization of tumor cells in mouse lung was monitored 24 hours after i.v. injection by bioluminescence and followed twice a week thereafter. To block TGF- $\beta$ in vivo, mice were treated with 2 G7 delivered i.p. at a dose of $15 \mathrm{mg} / \mathrm{kg}$ starting 24 hours before delivery of $10 \mathrm{~Gy}$ to mouse thorax and continued twice a week until death.

Bioluminescence imaging. To quantitate the bioluminescence signal as a measure of tumor burden, luciferin substrate (D-luciferin potassium salt; Promega) dissolved in deionized water was injected i.p. $(0.15 \mathrm{mg} / \mathrm{g}$ body weight) and images were acquired within 12 minutes of injection. According to our initial time course experiment, peak signal intensity was observed at that time (data not shown). Mice were anesthetized using isofluorane anesthesia during imaging. Bioluminescence imaging was performed by using the IVIS-200 imaging system (Xenogen) at the Vanderbilt University Small Animal Imaging Center. An integration time of 3-5 minutes was used with on-chip binning of 8 (in order to increase signal-to-noise ratio). Quantitative analysis of bioluminescence images was accomplished using 
the Living Image software (Xenogen) by defining regions of interest (ROI) and measuring integrated photon counts.

Statistics. All experimental data were analyzed using 2-tailed Student's $t$ test. A $P$ value less than 0.05 was considered significant.

\section{Acknowledgments}

This work was supported by NIH grants R01 CA85492 and R01 CA102162 (to H.L. Moses), R01 CA38079 (to M.L. Freeman), and R01 CA62212 (to C.L. Arteaga); by a grant from Aventis (to C.L. Arteaga); by Breast Cancer Specialized Program of Research Excel- lence (SPORE) grant P50 CA98131; and by Vanderbilt-Ingram Cancer Center Support Grant P30 CA68485.

Received for publication October 25, 2006, and accepted in revised form February 6, 2007.

Address correspondence to: Carlos L. Arteaga, Division of Oncology, Vanderbilt University Medical Center, 2220 Pierce Avenue, 777 PRB, Nashville, Tennessee 37232-6307, USA. Phone: (615) 936-3524; Fax: (615) 936-1790; E-mail: carlos.arteaga@vanderbilt.edu.
1. Massague, J., Seoane, J., and Wotton, D. 2005. Smad transcription factors. Genes Dev. 19:2783-2810.

2. Siegel, P.M., and Massague, J. 2003. Cytostatic and apoptotic actions of TGF-beta in homeostasis and cancer. Nat. Rev. Cancer. 3:807-820.

3. Bierie, B., and Moses, H.L. 2006. Tumour microenvironment: TGFbeta: the molecular Jekyll and Hyde of cancer. Nat. Rev. Cancer. 6:506-520.

4. Dumont, N., and Arteaga, C.L. 2003. Targeting the TGF beta signaling network in human neoplasia. Cancer Cell. 3:531-536.

5. Wakefield, L.M., and Roberts, A.B. 2002. TGF-beta signaling: positive and negative effects on tumorigenesis. Curr. Opin. Genet. Dev. 12:22-29.

6. Arteaga, C.L. 2006. Inhibition of TGFbeta signaling in cancer therapy. Curr. Opin. Genet. Dev. 16:30-37.

7. Derynck, R., and Zhang, Y.E. 2003. Smad-dependent and Smad-independent pathways in TGF-beta family signalling. Nature. 425:577-584.

8. Shin, I., Bakin, A.V., Rodeck, U., Brunet, A., and Arteaga, C.L. 2001. Transforming growth factor beta enhances epithelial cell survival via Aktdependent regulation of FKHRL1. Mol. Biol. Cell. 12:3328-3339.

9. Huang, Y., et al. 2000. Transforming growth factorbeta 1 suppresses serum deprivation-induced death of A549 cells through differential effects on c-Jun and JNK activities. J. Biol. Chem. 275:18234-18242.

10. Muraoka-Cook, R.S., et al. 2004. Conditional overexpression of active transforming growth factor beta1 in vivo accelerates metastases of transgenic mammary tumors. Cancer Res. 64:9002-9011.

11. Yi, J.Y., Shin, I., and Arteaga, C.L. 2005. Type I transforming growth factor beta receptor binds to and activates phosphatidylinositol 3-kinase. J. Biol. Chem. 280:10870-10876.

12. Muraoka-Cook, R.S., et al. 2006. Activated type I TGFbeta receptor kinase enhances the survival of mammary epithelial cells and accelerates tumor progression. Oncogene. 25:3408-3423.

13. Liu, P., Menon, K., Alvarez, E., Lu, K., and Teicher, B.A. 2000. Transforming growth factor-beta and response to anticancer therapies in human liver and gastric tumors in vitro and in vivo. Int. J. Oncol. 16:599-610.

14. Teicher, B.A., Kakeji, Y., Ara, G., Herbst, R.S., and Northey, D. 1997. Prostate carcinoma response to cytotoxic therapy: in vivo resistance. In Vivo. 11:453-461.

15. Teicher, B.A., Ikebe, M., Ara, G., Keyes, S.R., and Herbst, R.S. 1997. Transforming growth factorbeta 1 overexpression produces drug resistance in vivo: reversal by decorin. In Vivo. 11:463-472.

16. Wojtowicz-Praga, S. 2003. Reversal of tumorinduced immunosuppression with TGF-beta inhibitors. Invest. New Drugs. 21:21-32.

17. Ito, N., et al. 1995. Positive correlation of plasma transforming growth factor-beta 1 levels with tumor vascularity in hepatocellular carcinoma. Cancer Lett. 89:45-48.

18. Shariat, S.F., et al. 2001. Preoperative plasma levels of transforming growth factor beta(1) strongly predict clinical outcome in patients with bladder

\section{carcinoma. Cancer. 92:2985-2992.}

19. Shariat, S.F., et al. 2001. Preoperative plasma levels of transforming growth factor beta(1) (TGFbeta(1)) strongly predict progression in patients undergoing radical prostatectomy. J. Clin. Oncol. 19:2856-2864.

20. Tsushima, H., et al. 2001. Circulating transforming growth factor beta 1 as a predictor of liver metastasis after resection in colorectal cancer. Clin. Cancer Res. 7:1258-1262.

21. Rich, J.N. 2003. The role of transforming growth factor-beta in primary brain tumors. Front. Biosci. 8:e245-e260

22. Siegel, P.M., Shu, W., Cardiff, R.D., Muller, W.J., and Massague, J. 2003. Transforming growth factor beta signaling impairs Neu-induced mammary tumorigenesis while promoting pulmonary metastasis. Proc. Natl. Acad. Sci. U. S. A. 100:8430-8435.

23. Muraoka, R.S., et al. 2002. Blockade of TGF- $\beta$ inhibits mammary tumor cell viability, migration, and metastases. J. Clin. Invest. 109:1551-1559. doi: 10.1172/JCI200215234.

24. Muraoka, R.S., et al. 2003. Increased malignancy of Neu-induced mammary tumors overexpressing active transforming growth factor beta1. Mol. Cell. Biol. 23:8691-8703.

25. Anscher, M.S., Peters, W.P., Reisenbichler, H., Petros, W.P., and Jirtle, R.L. 1993. Transforming growth factor beta as a predictor of liver and lung fibrosis after autologous bone marrow transplantation for advanced breast cancer. N. Engl. J. Med. 328:1592-1598.

26. Kakeji, Y., Maehara, Y., Ikebe, M., and Teicher, B.A. 1997. Dynamics of tumor oxygenation, CD31 staining and transforming growth factor-beta levels after treatment with radiation or cyclophosphamide in the rat 13762 mammary carcinoma. Int. J. Radiat. Oncol. Biol. Phys. 37:1115-1123.

27. Barcellos-Hoff, M.H., Derynck, R., Tsang, M.L., and Weatherbee, J.A. 1994. Transforming growth factor- $\beta$ activation in irradiated murine mammary gland. J. Clin. Invest. 93:892-899.

28. Vujaskovic, Z., Marks, L.B., and Anscher, M.S. 2000. The physical parameters and molecular events associated with radiation-induced lung toxicity. Semin. Radiat. Oncol. 10:296-307.

29. Abe, M., et al. 1994. An assay for transforming growth factor-beta using cells transfected with a plasminogen activator inhibitor-1 promoter-luciferase construct. Anal. Biochem. 216:276-284.

30. Lucas, C., et al. 1990. The autocrine production of transforming growth factor-beta 1 during lymphocyte activation. A study with a monoclonal antibody-based ELISA. J. Immunol. 145:1415-1422.

31. Arteaga, C.L., Carty-Dugger, T., Moses, H.L., Hurd, S.D., and Pietenpol, J.A. 1993. Transforming growth factor beta 1 can induce estrogen-independent tumorigenicity of human breast cancer cells in athymic mice. Cell Growth Differ. 4:193-201.

32. Arteaga, C.L., et al. 1993. Anti-transforming growth factor (TGF)- $\beta$ antibodies inhibit breast cancer cell tumorigenicity and increase mouse spleen natural killer cell activity. Implications for a possible role of tumor cell/host TGF- $\beta$ interactions in human breast cancer progression. J. Clin. Invest. 92:2569-2576.

33. Forrester, E., et al. 2005. Effect of conditional knockout of the type II TGF-beta receptor gene in mammary epithelia on mammary gland development and polyomavirus middle $\mathrm{T}$ antigen induced tumor formation and metastasis. Cancer Res. 65:2296-2302.

34. Oft, M., Akhurst, R.J., and Balmain, A. 2002. Metastasis is driven by sequential elevation of $\mathrm{H}$-ras and Smad2 levels. Nat. Cell Biol. 4:487-494.

35. Tian, F., et al. 2004. Smad-binding defective mutant of transforming growth factor beta type I receptor enhances tumorigenesis but suppresses metastasis of breast cancer cell lines. Cancer Res. 64:4523-4530.

36. Ventura, J.J., Kennedy, N.J., Flavell, R.A., and Davis, R.J. 2004. JNK regulates autocrine expression of TGF-beta1. Mol. Cell. 15:269-278.

37. Kim, J.J., and Tannock, I.F. 2005. Repopulation of cancer cells during therapy: an important cause of treatment failure. Nat. Rev. Cancer. 5:516-525.

38. Anscher, M.S., Thrasher, B., Rabbani, Z., Teicher, B., and Vujaskovic, Z. 2006. Antitransforming growth factor-beta antibody 1D11 ameliorates normal tissue damage caused by high-dose radiation. Int. J. Radiat. Oncol. Biol. Phys. 65:876-881.

39. Hofer, S.O., et al. 1999. The effect of surgical wounding on tumour development. Eur. J. Surg. Oncol. 25:231-243.

40. Yamaguchi, K., Takagi, Y., Aoki, S., Futamura, M., and Saji, S. 2000. Significant detection of circulating cancer cells in the blood by reverse transcriptase-polymerase chain reaction during colorectal cancer resection. Ann. Surg. 232:58-65.

41. Shim, K.S., Kim, K.H., Han, W.S., and Park, E.B. 1999. Elevated serum levels of transforming growth factor-beta 1 in patients with colorectal carcinoma: its association with tumor progression and its significant decrease after curative surgical resection. Cancer. 85:554-561.

42. Feltl, D., Zavadova, E., Pala, M., and Hozak, P. 2005. The dynamics of plasma transforming growth factor beta 1 (TGF-beta1) level during radiotherapy with or without simultaneous chemotherapy in advanced head and neck cancer. Oral Oncol. 41:208-213.

43. Robert, F., et al. 2003. Phase II study of docetaxel plus enoxaparin in chemotherapy-naive patients with metastatic non-small cell lung cancer: preliminary results. Lung Cancer. 42:237-245.

44. Chytil, A., Magnuson, M.A., Wright, C.V., and Moses, H.L. 2002. Conditional inactivation of the TGF-beta type II receptor using Cre:Lox. Genesis. 32:73-75.

45. Wyckoff, J.B., Jones, J.G., Condeelis, J.S., and Segall, J.E. 2000. A critical step in metastasis: in vivo analysis of intravasation at the primary tumor. Cancer Res. 60:2504-2511.

46. Wang, S.E., Wu, F.Y., Shin, I., Qu, S., and Arteaga, C.L. 2005. Transforming growth factor beta (TGF-beta)-Smad target gene protein tyrosine phosphatase receptor type kappa is required for TGF-beta function. Mol. Cell. Biol. 25:4703-4715. 